Learning scenarios

\begin{tabular}{|c|c|c|}
\hline Scenario & Task & Score \\
\hline Scenario 1 & & \\
\hline $\begin{array}{l}\text { Image used: } \\
\text { X-ray of } \\
\text { elbow } \\
\text { frontal/profile }\end{array}$ & & \\
\hline \multirow[t]{6}{*}{$\begin{array}{l}\text { Starting } \\
\text { point: X-ray } \\
\text { frontal view }\end{array}$} & $\begin{array}{l}\text { Open } \\
\text { session } \\
\text { (hand } \\
\text { waving } \\
\text { like in all } \\
\text { following } \\
\text { session } \\
\text { openings) }\end{array}$ & 0 \\
\hline & $\begin{array}{l}\text { Zoom on } \\
\text { elbow: }\end{array}$ & \\
\hline & Selection & 1 \\
\hline & Utilization & 1 \\
\hline & Quit & 1 \\
\hline & $\begin{array}{l}\text { Close } \\
\text { session }\end{array}$ & 0 \\
\hline Max. score & & 3 \\
\hline
\end{tabular}

Scenario 2

Image used:

X-ray of

elbow

frontal/profile

\begin{tabular}{lll}
\hline Starting & Open & 0 \\
point: X-ray & session & \\
profile & & \\
& &
\end{tabular}

\begin{tabular}{ll}
\hline Zoom in & \\
on image: & \\
Selection & 1 \\
Utilization & 1 \\
Quit & 1 \\
\hline Increase & \\
contrast: & \\
Selection & 1 \\
Utilization & 1 \\
Quit & 1 \\
\hline Reset & \\
view: & \\
Utilization & 1 \\
& \\
\hline Close & 0 \\
session &
\end{tabular}

Scenario 3

Image used:

X-ray of

elbow

frontal/profile

\begin{tabular}{lll}
\hline Starting & Open & 0 \\
point: X-ray & session & \\
profile view & &
\end{tabular}

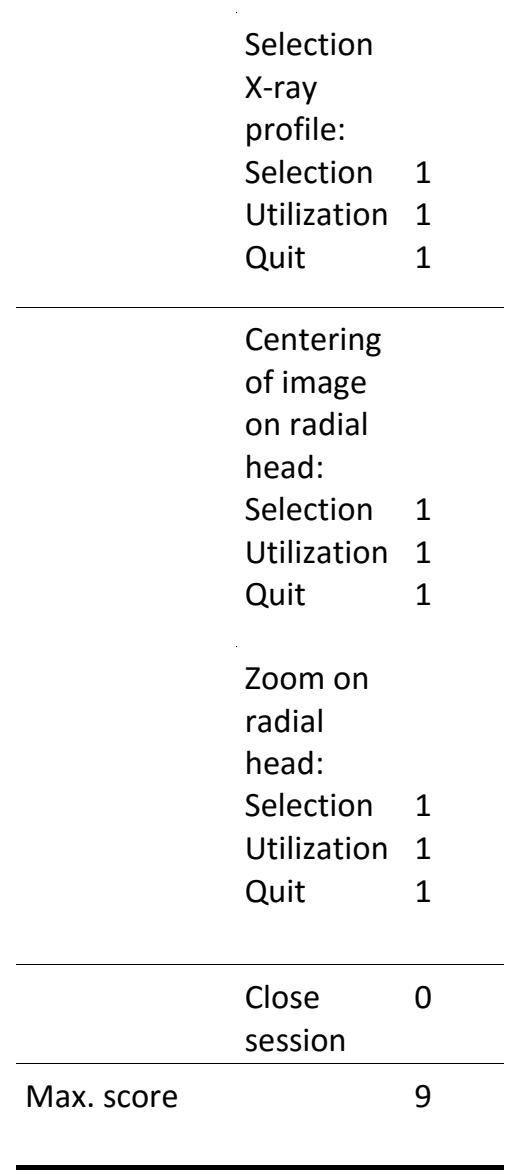

\section{Scenario 4}

Image used:

$X$-ray of

elbow

frontal/profile

$+\mathrm{CT}$ elbow

\begin{tabular}{|c|c|}
\hline $\begin{array}{l}\text { Starting } \\
\text { point: X-ray }\end{array}$ & $\begin{array}{l}\text { Open } \\
\text { session }\end{array}$ \\
\hline
\end{tabular}

Add

sagittal

view of

CT:

Selection 1

Utilization 1

Quit 1
Scroll to

the

fracture of

radial

head:

Selection 1

Utilization 1

Quit 1

Split

screen

vertically:

Selection 1

Utilization 1

Quit 1

Add image

X-ray

profile:

Selection 1

Utilization 1

Quit

1

\begin{tabular}{lll}
\hline & $\begin{array}{l}\text { Close } \\
\text { session }\end{array}$ & 0 \\
\hline Max. score & 12 \\
\hline
\end{tabular}

Scenario 5

Image used:

X-ray of

elbow

frontal/profile

+ CT elbow

\begin{tabular}{lll}
\hline Starting & Open & 0 \\
point: CT 3D & session &
\end{tabular}

Rotate CT

in $3 \mathrm{D}(360$

deg.):

Selection 1

Utilization 1

Quit 1

Add X-ray

profile of

elbow

Selection 1

Utilization 1

Quit 1

Zoom on

olecranon:

Selection 1

Utilization 1

Quit 1 


\begin{tabular}{|c|c|c|c|c|c|}
\hline & $\begin{array}{l}\text { Point on } \\
\text { olecranon: } \\
\text { Selection } \\
\text { Utilization } \\
\text { Quit }\end{array}$ & $\begin{array}{l}1 \\
1 \\
1\end{array}$ & $\begin{array}{l}\text { Starting } \\
\text { point: CT } \\
\text { with sagittal } \\
\text { slice }\end{array}$ & $\begin{array}{l}\text { Open } \\
\text { session }\end{array}$ & 0 \\
\hline & & & & $\begin{array}{l}\text { Split } \\
\text { screen }\end{array}$ & \\
\hline & $\begin{array}{l}\text { Close } \\
\text { session }\end{array}$ & 0 & & $\begin{array}{l}\text { vertically: } \\
\text { Selection }\end{array}$ & 1 \\
\hline Max. score & & 12 & & Utilization & 1 \\
\hline & & & & Quit & 1 \\
\hline Test scena & rios & & & $\begin{array}{l}\text { Add } \\
\text { coronal }\end{array}$ & \\
\hline Scenario & Task & Score & & the right & \\
\hline Scenario 1 & & & & $\begin{array}{l}\text { pane: } \\
\text { Selection }\end{array}$ & 1 \\
\hline CT of elbow & & & & Utilization & 1 \\
\hline $\begin{array}{l}\text { with coronal, } \\
\text { axial slices }\end{array}$ & & & & Quit & 1 \\
\hline and 3D & & & & $\begin{array}{l}\text { Increase } \\
\text { contrast: }\end{array}$ & \\
\hline $\begin{array}{l}\text { Starting } \\
\text { point: } \mathrm{CT} \\
\text { sagittal slide }\end{array}$ & $\begin{array}{l}\text { Open } \\
\text { session } \\
\text { (hand }\end{array}$ & 0 & & $\begin{array}{l}\text { Selection } \\
\text { Utilization } \\
\text { Quit }\end{array}$ & $\begin{array}{l}1 \\
1 \\
1\end{array}$ \\
\hline & $\begin{array}{l}\text { waving } \\
\text { like in all } \\
\text { following } \\
\text { session }\end{array}$ & & & $\begin{array}{l}\text { Close } \\
\text { session }\end{array}$ & 0 \\
\hline & openings) & & Max. Score & & 9 \\
\hline & $\begin{array}{l}\text { Scroll to } \\
\text { fracture of } \\
\text { ulna } \\
\text { Selection } \\
\text { Utilization } \\
\text { Quit }\end{array}$ & $\begin{array}{l}1 \\
1 \\
1\end{array}$ & $\begin{array}{l}\text { Scenario } 3 \\
\text { Image used: } \\
\text { CT of elbow } \\
\text { with coronal, } \\
\text { axial slices }\end{array}$ & & \\
\hline & $\begin{array}{l}\text { Zoom on } \\
\text { fracture: }\end{array}$ & & and 3D & & \\
\hline & Selection & 1 & Starting & Open & 0 \\
\hline & Utilization & 1 & point: CT & session & \\
\hline & Quit & 1 & $\begin{array}{l}\text { with sagittal } \\
\text { slice }\end{array}$ & & \\
\hline & Center on & & & & \\
\hline & olecrane & & & Selection & \\
\hline & Selection & 1 & & 3D recon- & \\
\hline & Utilization & 1 & & struction: & \\
\hline & Quit & 1 & & Selection & 1 \\
\hline & & & & Utilization & 1 \\
\hline & Close & 0 & & Quit & 1 \\
\hline & session & & & Rotate to & \\
\hline Max. score & & 9 & & $\begin{array}{l}\text { frontal } \\
\text { view of }\end{array}$ & \\
\hline Scenario 2 & & & & $\begin{array}{l}\text { elbow: } \\
\text { Selection }\end{array}$ & 1 \\
\hline Image used: & & & & Utilization & 1 \\
\hline $\begin{array}{l}\text { CT with } \\
\text { coronal, axial } \\
\text { slices and 3D }\end{array}$ & & & & Quit & 1 \\
\hline
\end{tabular}

\begin{tabular}{|c|c|c|}
\hline & $\begin{array}{l}\text { Zoom on } \\
\text { elbow: } \\
\text { Selection } \\
\text { Utilization } \\
\text { Quit }\end{array}$ & $\begin{array}{l}1 \\
1 \\
1\end{array}$ \\
\hline & $\begin{array}{l}\text { Close } \\
\text { session }\end{array}$ & 0 \\
\hline Max. score & & 7 \\
\hline $\begin{array}{l}\text { Scenario } 4 \\
\text { Image used: } \\
\text { X-ray of knee } \\
\text { frontal and } \\
\text { profile }\end{array}$ & & \\
\hline \multirow[t]{4}{*}{$\begin{array}{l}\text { Starting } \\
\text { point: X-ray } \\
\text { profile }\end{array}$} & $\begin{array}{l}\text { Open } \\
\text { session }\end{array}$ & 0 \\
\hline & $\begin{array}{l}\text { Point to } \\
\text { patella: } \\
\text { Selection } \\
\text { Utilization } \\
\text { Quit } \\
\text { Center on } \\
\text { letter D in } \\
\text { image: } \\
\text { Selection } \\
\text { Utilization } \\
\text { Quit }\end{array}$ & $\begin{array}{l}1 \\
1 \\
1\end{array}$ \\
\hline & $\begin{array}{l}\text { Open } \\
\text { frontal X- } \\
\text { ray: } \\
\text { Selection } \\
\text { Utilization } \\
\text { Quit }\end{array}$ & $\begin{array}{l}1 \\
1 \\
1\end{array}$ \\
\hline & $\begin{array}{l}\text { Close } \\
\text { session }\end{array}$ & 0 \\
\hline Max. score & & 9 \\
\hline Scenario 5 & & \\
\hline $\begin{array}{l}\text { Image used: } \\
\text { X-ray of knee } \\
\text { frontal and } \\
\text { profile }\end{array}$ & & \\
\hline $\begin{array}{l}\text { Starting } \\
\text { point: X-ray } \\
\text { frontal }\end{array}$ & $\begin{array}{l}\text { Open } \\
\text { session }\end{array}$ & 0 \\
\hline
\end{tabular}




\begin{tabular}{|c|c|c|}
\hline & $\begin{array}{l}\text { Split } \\
\text { screen } \\
\text { vertically } \\
\text { Selection } \\
\text { Utilization } \\
\text { Quit }\end{array}$ & $\begin{array}{l}1 \\
1 \\
1\end{array}$ \\
\hline & $\begin{array}{l}\text { Add X-ray } \\
\text { profile } \\
\text { Selection } \\
\text { Utilization } \\
\text { Quit }\end{array}$ & $\begin{array}{l}1 \\
1 \\
1\end{array}$ \\
\hline & $\begin{array}{l}\text { Modify } \\
\text { contrast } \\
\text { of X-ray } \\
\text { profile: } \\
\text { Selection } \\
\text { Utilization } \\
\text { Quit }\end{array}$ & $\begin{array}{l}1 \\
1 \\
1\end{array}$ \\
\hline & $\begin{array}{l}\text { Close } \\
\text { session }\end{array}$ & 0 \\
\hline Max. score & & 9 \\
\hline
\end{tabular}

\section{Scenario 6}

Image used:

X-ray of knee

frontal and

profile

\begin{tabular}{lll}
\hline $\begin{array}{l}\text { Starting } \\
\text { point: X-ray } \\
\text { frontal }\end{array}$ & $\begin{array}{l}\text { Open } \\
\text { session }\end{array}$ & 0 \\
\hline & Zoom & \\
& Selection & 1 \\
& Utilization & 1 \\
& Quit & 1
\end{tabular}

\begin{tabular}{lll}
\hline & Modify & \\
& contrast: & \\
& Selection & 1 \\
& Utilization & 1 \\
& Quit & 1 \\
& & \\
& Reset & \\
& image: & \\
& Utilization & 1 \\
& & \\
& & \\
& & \\
\hline Max. score & & \\
& Close & 0 \\
\hline & \\
\hline
\end{tabular}

\section{Scenario 7}

Image used:

X-ray of

elbow and CT

\begin{tabular}{|c|c|c|}
\hline $\begin{array}{l}\text { Starting } \\
\text { point: X-ray } \\
\text { profile }\end{array}$ & $\begin{array}{l}\text { Open } \\
\text { session }\end{array}$ & 0 \\
\hline & $\begin{array}{l}\text { Point on } \\
\text { fracture: } \\
\text { Selection } \\
\text { Utilization } \\
\text { Quit }\end{array}$ & $\begin{array}{l}1 \\
1 \\
1\end{array}$ \\
\hline & $\begin{array}{l}\text { Center on } \\
\text { letter D: } \\
\text { Selection } \\
\text { Utilization } \\
\text { Quit }\end{array}$ & $\begin{array}{l}1 \\
1 \\
1\end{array}$ \\
\hline & $\begin{array}{l}\text { Add X-ray } \\
\text { frontal: } \\
\text { Selection } \\
\text { Utilization } \\
\text { Quit }\end{array}$ & $\begin{array}{l}1 \\
1 \\
1\end{array}$ \\
\hline & $\begin{array}{l}\text { Close } \\
\text { session }\end{array}$ & 0 \\
\hline Max. score & & 9 \\
\hline
\end{tabular}

\section{Scenario 8}

Image used:

X-ray elbow

and $\mathrm{CT}$

\begin{tabular}{lll}
\hline $\begin{array}{l}\text { Starting } \\
\text { point: X-ray } \\
\text { frontal }\end{array}$ & $\begin{array}{l}\text { Open } \\
\text { session }\end{array}$ & 0 \\
\hline & Zoom on & \\
& elbow & \\
& Selection & 1 \\
& Utilization & 1 \\
& Quit & 1
\end{tabular}

Decrease

contrast,

making $X$ -

ray more

grayish

Selection 1

Utilization 1

Quit 1
Reset

image:

Utilization 1

\begin{tabular}{lll}
\hline & $\begin{array}{l}\text { Close } \\
\text { session }\end{array}$ & 0 \\
\hline Max. score & & 9 \\
\hline
\end{tabular}

\section{Scenario 9}

Image used:

X-ray profile

of elbow and

CT 3D

\begin{tabular}{lll}
\hline Starting & Open & 0 \\
point: CT 3D & session &
\end{tabular}

Rotate

image

(360 deg.)

Selection 1

Utilization 1

Quit 1

Zoom

Selection 1

Utilization 1

Quit 1

Add X-ray

profile:

Selection 1

Utilization 1

Quit 1

\begin{tabular}{lll}
\hline & $\begin{array}{l}\text { Close } \\
\text { session }\end{array}$ & 0 \\
\hline Max. score & & 9 \\
\hline
\end{tabular}

